Archives de sciences sociales des religions

111 | juillet-septembre 2000

Varia

\title{
Le Sensible incontournable
}

Rites et refus du ritualisme au sein de l'Église Réformée

\section{Françoise Lautman}

\section{(2) OpenEdition}

\section{Journals}

Édition électronique

URL : http://journals.openedition.org/assr/20227

DOI : $10.4000 /$ assr.20227

ISSN : 1777-5825

Éditeur

Éditions de l'EHESS

\section{Édition imprimée}

Date de publication : 1 septembre 2000

Pagination : 95-111

ISBN : 2-222-96695-7

ISSN : 0335-5985

\section{Référence électronique}

Françoise Lautman, « Le Sensible incontournable », Archives de sciences sociales des religions [En

ligne], 111 | juillet-septembre 2000, mis en ligne le 19 août 2009, consulté le 30 avril 2019. URL : http:// journals.openedition.org/assr/20227 ; DOI : 10.4000/assr.20227

Ce document a été généré automatiquement le 30 avril 2019.

(c) Archives de sciences sociales des religions 


\title{
Le Sensible incontournable
}

\author{
Rites et refus du ritualisme au sein de l'Église Réformée
}

Françoise Lautman

1 Les formes des cultes et de la Cène ont été dès les premiers temps de la Réforme inspirées par deux principes : - une liturgie simple dont la compréhension soit accessible à tous, la participation de tous à son déroulement. Les grandes lignes vont s'en établir assez rapidement, par élagage progressif de tout ce qui paraissait magisme, mystère et spectacle dans la messe tout en en conservant la structure. L'essentiel est fait entre 1524, première messe en langue commune en Alsace, et la publication par Calvin à Genève en $1542^{1}$ de $\mathrm{La}$ forme des prières et chants ecclésiastiques, avec la manière d'administrer les sacrements et consacrer le mariage selon la coutume de l'Église ancienne. C'est ce texte maintes fois réédité qui constitue la tradition cultuelle de presque toutes les Églises Réformées. L'universalité de cette référence, l'invocation de l'Église ancienne, la simplicité recherchée auraient pu être autant de gages d'unité. En fait, dès les premiers temps également, c'est la diversité qui a prévalu en raison des mêmes principes. Ils impliquent la liberté de chaque église locale d'adopter les formes qui conviennent le mieux à la compréhension et à la participation de tous ses membres.

2 Les lignes de force du culte réformé et de la Cène demeurent actuellement celles instituées par les réformateurs : emploi exclusif de la langue vivante, place centrale des lectures bibliques et de la prédication, chants par toute l'assemblée avec large place faite aux psaumes, communion de tous sous les deux espèces.

Dans cette même revue, il y a quelques années, Bernard Roussel ${ }^{2}$ dénonçait l'illusion qu'il y a à dire devant la sobriété, «l'absence quasi-totale de pratiques aberrantes ou jugées telles... des rites réformés : passez votre chemin, il n'y a rien à voir!» alors que le protestantisme est comme toute religion, «construit, entretenu, transmis par des rites ». Il incitait historiens des religions et anthropologues à "trouver matière à analyse et réflexion dans l'observation du rite de la Cène, tel que le pratiquent les réformés français $\mathrm{du} \mathrm{XVI}^{\mathrm{e}}$ siècle ». C'est ce que nous entendons essayer auprès de nos contemporains sans prétendre en épuiser la matière. L'intellectualisme, la sécheresse, qu'on reproche toujours actuellement aux réformés français nous a également paru relever de l'idée 
reçue, y compris de leur propre fait, tant elle semble cohérente avec le rejet de toute compromission avec le magisme ou simplement l'émotionnel.

La liturgie de l'Église Réformée de France est actuellement au carrefour de plusieurs lignes d'évolution. Lorsqu'elle s'est constituée en 1938 en regroupant quatre Unions d'Églises, chacune avait ses propres traditions liturgiques. Il fallut, et il faut encore, concilier le respect des traditions locales avec la recherche d'unité propre à l'Église. La confrontation avec les rites catholiques se pose aujourd'hui en des termes différents aussi bien en raison de la perte de son emprise sur la culture commune que dans un contexte favorable à l'oecuménisme. Enfin, la recherche théologique met de plus en plus l'accent sur l'importance des rites, de la corporalité, du rapport au symbolique... Il sera fait allusion à ces évolutions-là où nous en rencontrerons la recherche ou les effets mais ce n'est pas notre objet d'en faire l'historique, même brièvement ${ }^{3}$.

5 L'observateur extérieur qui pénètre, dimanche après dimanche, dans les temples parisiens ou de régions diverses de l'Église Réformée de France (ERF) est d'abord frappé par la simplicité, pourrait-on dire familière, des façons de faire à l'intérieur de ce cadre donné mais aussi par leur variété. Il l'est d'autant plus peut-être que le contexte culturel français, catholique, a contribué à une conception du rite religieux comme fixant gestes et paroles dans les moindres détails, conditions de son efficacité et garanties de son identité à travers les âges et les diversités nationales ${ }^{4}$. Ce qu'il y a à voir justement, c'est l'usage du rite propre à cette communauté protestante particulière, mais aussi de son contexte, les lieux, les heures, les gestes qui sans en faire partie lui sont liés par la coutume ou par la simple commodité. Dans le cadre d'une réflexion sur les formes sensibles de la religion, il a semblé intéressant de recenser ces façons de faire qui portent sur des aspects très concrets mais allant de ce qui est reconnu le plus central, les pratiques concernant la prédication et la Cène $\mathrm{e}^{5}$, à ce qui peut sembler le plus accessoire et d'évaluer si les acteurs leur reconnaissent ou non de l'importance, de quelle façon ces pratiques sont perçues, les implications qu'elles peuvent avoir sur le sens reconnu aux rites... enfin de les mettre éventuellement en rapport avec les récents renouvellements de la liturgie de l'ERF.

\section{Formes et lieux}

6 L'observation systématique de cultes dominicaux et de Cènes, des gestes les plus rituels aux plus simplement coutumiers ou apparemment dépourvus d'importance religieuse, a été conduite au cours des années 1995 à 1998 à Paris ${ }^{6}$, en Limousin ${ }^{7}$, en Normandie ${ }^{8}$, en Languedoc $^{9}$ et en Provence ${ }^{10}$. Lieux, ordre du culte, importance de l'assistance, chants, thème de la prédication, gestes de l'officiant et des participants étaient portés en notes manuscrites aussi détaillées que possible au cours même du culte. Ces observations ont ensuite été résumées sous forme de fiches. Nous en présentons ici deux à titre d'exemple.

\section{$1^{\text {re }}$ observation}

Le dimanche de Pentecôte dans une région à faible population protestante :

Le lieu:

Un temple ancien, sans ornement, trop vaste pour une communauté actuellement réduite à une soixantaine de personnes dont dix seulement (d'âge relativement élevé) sont plus ou moins régulièrement présentes au culte, quarante-cinq ce jour-là dont un groupe de visiteurs (également réformés); disposition concentrique des bancs autour d'une table de 
communion quand l'assistance est réduite, devant la table quand elle est plus nombreuse comme ici ; fenêtres de verre blanc dépoli ; chaire élevée derrière la table de communion, orgue lui faisant face; sur la table coupes et plat d'argent, contenant vin rouge et pain prédécoupé, sont couverts par des voiles; Bible ouverte face au public; tableaux face à l'assemblée portant les chiffres des cantiques du jour.

Le culte:

Pasteur en robe noire et rabats blancs

Accueil de l'assemblée

Invocation

Ps 100 st 3. «Présentez-vous tous devant lui »

Proclamation de la loi

Ps 117 « Vous tous les peuples, louez Dieu » (le pasteur a oublié la référence, il demande à un conseiller de la lui rappeler)

Confession des péchés

Ps 139 st 4. « Partout, Seigneur, tu me conduis »

Annonce du pardon

Chant 190 st 3. «O Toi, Jésus crucifié » (le pasteur interpelle l'assistance puis l'organiste pour ajouter une strophe)

Confession de foi chantée « Dès que ma foi t'a connu et servi »

Chant (la chorale chante 2 motets de Bach)

Lectures bibliques (Actes 2, 20 et Rom. 1, 8)

Chant 748 st 1-2 « Le Seigneur seul est ma lumière »

Prédication (thème sans référence à la Pentecôte... le pasteur boit plusieurs fois en chaire pour s'éclaircir la voix)

Orgue

Annonces locales

Sainte Cène :

Récit de l'institution

(L'assemblée est invitée à faire deux cercles successivement autour de la table).

Le pasteur découvre le pain et en prend un morceau qu'il élève et rompt et le donne aux deux conseillers presbytéraux qui vont le distribuer. Il communie en premier avec eux.

Puis il découvre les coupes, en élève une, « voici la coupe de la nouvelle alliance... » et les propose aux conseillers à sa droite et à sa gauche qui les proposent ensuite à chacun. (Les coupes sont essuyées après chaque personne. Entre les deux cercles de communiants, la quantité prévue étant insuffisante, on retourne remplir les coupes à la sacristie. L'orgue ayant prévu un morceau trop court pour une participation à la Cène plus nombreuse que d'habitude, le pasteur l'interpelle pendant que les coupes circulent pour lui dire de continuer)

Prière d'intercession, Notre Père

Chant

Exhortation (et invitation à partager ensuite un café), bénédiction

Chant 212 st 3. 
Orgue

(Le pasteur salue individuellement chacun à la sortie)

\section{$2^{\mathrm{e}}$ observation}

Le dimanche des Rameaux dans un temple parisien avec participation des enfants de l'école biblique et baptême :

Le lieu:

Un temple relativement petit, 250 places environ, habituellement plein, population d'âges divers; bancs au centre, chaises sur les côtés; salle nue ornée seulement d'une grande croix latine en bois surmontant la chaire qui surplombe elle-même une grande table de bois nue sur laquelle sont posés deux plats de pain de mie coupé en dés, 4 coupes de métal argenté remplies de vin rouge, une cruche de même métal pour renouveler les coupes (une autre contient l'eau pour le baptême) et un cierge allumé à chaque extrémité ; à gauche de la table, le lutrin supportant une grande Bible ouverte face à l'assemblée, et sur l'autre face les documents du pasteur; à droite un grand bouquet de fleurs; tableaux indiquant les numéros des chants de ce jour de chaque côté de la chaire; fenêtres en vitrail à motifs géométriques où domine le blanc; orgue au fond de la chapelle, faisant face à la chaire.

\section{Le culte:}

Pasteur en robe noire et rabats blancs

Orgue

Accueil

(Entrée en procession des enfants des trois groupes bibliques portant des rameaux et chantant Alléluia! Ils se dirigent directement par erreur vers le pasteur qui les fait ressortir et recommencer leur entrée en prenant comme prévu l'allée centrale. Ils viennent déposer leurs rameaux devant la table de communion et s'asseyent sur les premiers bancs.)

Ps 47 st 1.2.3 « Frappez dans vos mains »

Annonce de la Grâce, confession des péchés

Chant des enfants : «Écoutez tous, je vous supplie »

Proclamation du pardon

Ps 118 st 1. «Célébrez Dieu, rendez-lui grâce »

Confession de foi

Chant 471 st 1. «À toi la Gloire, ô Ressuscité »

Baptême d'un bébé. (Avant le baptême, les enfants de l'école biblique, regroupés devant la table, reçoivent tous dans la main une bougie allumée avec conseils de prudence. On leur explique que "Jésus est lumière » et que la bougie est une prière, puis on les souffle avant même que le baptême ne soit administré.

Pendant le baptême tout le monde est invité à se lever pour se manifester comme témoins.)

Chant 561 st 1.3. «Ô notre Dieu, Père tendre et fidèle »

Prière d'illumination

Lecture biblique : Matt. 21, 1-17

Chant 257 st 1.2.3.4.5. «Jésus, ton nom est le plus beau » 

absolument pas nécessaire d'être dans un temple pour faire un culte, n'importe quelle salle ou même un lieu extérieur peuvent théoriquement convenir. À s'en tenir toujours à l'Église Réformée de France, on rencontre une incontestable diversité architecturale des lieux, diversité due aussi bien à l'époque de la construction des temples qu'à la situation plus ou moins minoritaire du protestantisme selon les régions. Cette dernière a joué sur le souci de visibilité ou d'effacement ${ }^{11}$. La plupart des temples français ont été construits dans la première moitié du XIX ${ }^{e}$ siècle dans un style néo-classique quis'accordait avec le souci protestant de sobriété et la nécessité politique de discrétion mais il y eut aussi quelques cas, généralement plus tardifs, de construction néogothique et quelques réutilisations d'églises octroyées (rarement catholiques, quelquefois anglicanes surtout dans les régions touristiques : Nice, Mégève...).

Sans méconnaître ces différences architecturales, les lieux et leur décor présentent de nombreux éléments d'uniformité d'un temple à l'autre. On pourrait dire si on assimile le sensible à l'émotif qu'ils n'y concèdent rien : dépouillement d'un décor immuable et réduit à sa plus simple expression, impression de rigidité renforcée par l'alignement des 
bancs de bois préférés le plus souvent aux chaises, couleurs claires et froides, larges fenêtres de vitrail où le blanc domine. On y rencontre généralement l'application des principes que Michel Pastoureau reconnaît plus largement à l'esthétique protestante : « ... la couleur est fard, luxe, illusion. Elle est vaine parce qu'elle est matière; elle est dangereuse parce qu'elle détourne du vrai et du bien; elle est coupable parce qu'elle tente de séduire et de tromper; elle est gênante parce qu'elle empêche de reconnaître clairement les formes et les contours ${ }^{12}$. » À la différence des lieux de culte catholiques ou orthodoxes, aucun espace n'y est considéré comme sacré ou mystérieux, pas d'obscurité ou de lumières suggestives, pas d'odeurs non plus: ni encens, ni cierges, les rares bouquets eux-mêmes semblent sans parfums... rien d'autre qu'une irréprochable netteté. Là encore on peut reconnaître un trait de culture commune aux protestants, Bernard Reymond rappelle qu'ils ont été, dès le milieu du XIX ${ }^{\mathrm{e}}$ siècle, partisans des mouvements hygiénistes : «la netteté des corps appelle la netteté de l'âme. Il y a de vieilles affinités entre le protestantisme et la chasse à la saleté... et simultanément la chasse aux mauvaises odeurs. De tous les effluves possibles le protestantisme préfère celui de la propreté1 $^{13}$ »

80 Monochromie, éclairage et disposition, neutralité du décor visent cependant uniquement à ce que l'on puisse bien voir et entendre quel que soit l'endroit où l'on se trouve (position centrale de la table, position élevée de la chaire...), à ce qu'aucun élément étranger ne vienne distraire de ce qui est en jeu, à faciliter donc la compréhension et la participation de tous. Ces dispositions, comme la prescription d'utiliser la langue commune, remontent aux premiers temps de la Réforme. Mais la stabilité de ces éléments dans le temps et l'espace constitue en conséquence un conditionnement sensible fort qui permet immédiatement de réaliser que l'on se trouve dans un lieu protestant et même de le situer dans l'éventail des protestantismes: la présence d'une grande croix nue audessus de la chaire, seule concession au symbolique, ne fait pas l'unanimité ; il n'y aura pas de table de communion, par exemple, dans des Églises plus radicales (Quakers) qui ne connaissent pas de sacrements; les luthériens, par contre, concèdent beaucoup plus au décor, aux représentations visuelles qu'ils n'excluent pas dès lors que toute idolâtrie, c'est-à-dire que tout culte adressé à ces représentations, est écarté.

81 Autres signes forts : la robe pastorale et la chaire. La robe pastorale n'est pas un vêtement religieux, mais le signe de diplômes universitaires exigés pour accéder à une charge pastorale. Dans le courant des années qui ont suivi 1968, certains ont voulu supprimer un vêtement qui suggérait une autorité magistrale et adopter le simple vêtement de ville, comme ils entendaient renoncer pour les mêmes raisons à prêcher depuis la chaire à laquelle l'usage des micros retirait d'ailleurs sa principale utilité pratique. Ce qui revenait à en méconnaître la forte charge symbolique : toutes les prescriptions liturgiques que j'ai pu consulter insistent sur le fait que seules la lecture des textes bibliques et la prédication doivent être effectués de la chaire, le reste du culte (et de la Cène !) doit être conduit au pied de la chaire ${ }^{14}$. Dans les mêmes années, la recherche d'oecuménisme en a incité d'autres à adopter une aube blanche qui rapprochait des catholiques. On peut dire avec le recul que ces tentatives ont fait long feu. Les manifestations en demeurent minoritaires. Des innovations plus importantes comme l'arrivée de femmes dans le corps pastoral ont même contribué au maintien d'un vêtement qui dispense des choix... et des critiques vestimentaires. ce que des recherches récentes ont mis en évidence comme une crise actuelle de la 
prédication? «C'est par elle que l'Église protestante s'atteste comme institution dispensatrice de biens de salut objectifs et non simplement comme association volontaire de croyants $^{15}$ ». Mais Jean-Paul Willaime souligne justement que perçue comme l'élément essentiel du rite, elle court le risque d'être réduite à son accomplissement et de cristalliser sur elle, sinon le magisme lié à ce qui met les hommes en rapport avec la divinité, la fonction de faire communier le groupe dans son identité, ou encore d'être simplement un soutien psychologique. Des enquêtes conduites en Allemagne ou en France font état de ce que les fidèles interrogés à la sortie du culte sont nombreux à ne pas pouvoir dire sur quoi portait la prédication et s'accordent sur la proposition suivante « la prédication apporte consolation et aide pour la vie ${ }^{16} »$. Dans ce contexte, robe et chaire rappellent au contraire de façon sensible que la prédication est un enseignement.

Les caractères sensibles apparaissent de façon évidente lorsqu'il s'agit des attitudes corporelles des fidèles accompagnant les phases du culte. Dire la profession de foi debout contribue à l'affirmer; écouter la proclamation de la grâce, de la loi, recevoir la bénédiction debout manifestent peut-être $\mathrm{F}$ importance des dons reçus, mais on chante debout, on écoute prières, lectures bibliques et prédications assis sans aucune importance autre en apparence que de confort et cela se fait aussi avec la plus grande régularité. Ces usages semblaient-ils menacés? ou au contraire y attache-t-on de plus en plus d'importance ? Il faut noter que les textes de la nouvelle liturgie commune ${ }^{17}$ comportent de façon redoublée les indications concernant ces attitudes : - en italique entre les textes liturgiques « l'officiant(e) invite d'un geste l'assemblée à se lever "... « à s'asseoir », - par un trait grisé vertical en marge des passages où l'assemblée doit se tenir debout.

Ces indications sont d'autant plus remarquables que par ailleurs, le souci d'éviter la sacralisation des lieux se retrouve dans les comportements. On prend des notes ostensiblement pendant lecture et prédication, on sort sans se donner la peine de contourner l'assemblée si nécessaire... On se tient décemment, avec un certain recueillement avant et pendant le culte, mais, dès les derniers mots du culte prononcés, on s'y rejoint de groupe en groupe, comme dans n'importe quelle salle de réunion et ce d'autant plus que la coutume de saluer individuellement le pasteur devant l'unique issue entraine souvent attente et piétinements. Pas de voix basses alors ni de gestes contenus comme on peut le constater dans les églises catholiques, par exemple.

Le chant d'assemblée a été l'un des traits fondateurs du changement liturgique inauguré par la Réforme, il manifestait la participation active des fidèles au culte. Il semble d'ailleurs toujours présenter pour les participants une activité liée à peu près uniquement aux cultes, les occasions de chanter, et de chanter ensemble, étant rares pour les adultes dans la culture actuelle. La musique et les chants utilisés pendant les cultes prennent part à cette identification sensible du culte protestant par le patrimoine musical et hymnologique qui s'est constitué au cours des siècles, de la Réforme au temps du Réveil et à l'époque actuelle. Il y a là un remarquable ensemble d'harmonie et d'inspiration dont un musicologue parlerait mieux que moi. On y a récemment introduit dans un esprit oecuménique quelques chants issus de la réforme liturgique catholique, adoptés en particulier dans la région lyonnaise. Ils suscitent toujours certaines résistances ${ }^{18}$, mais ils semblent s'intégrer progressivement. Par contre, j'ai été témoin de la quasi impossibilité à intégrer la gamme et la sensibilité orthodoxes dont, après quelques essais infructueux, le pasteur a dû interrompre la tentative d'exécution au cours même d'un culte.

86 Cependant la pratique concernant ce patrimoine musical est étonnante. C'est ce trait d'ailleurs qui m'a, au début, incitée à l'observation des formes sensibles du culte 
protestant. J'avais sollicité des enregistrements dans l'intention d'une présentation comparée des traditions de chants religieux juifs, catholiques et protestants, au cours des cultes hebdomadaires. La bande fournie pour le protestantisme a paru si mauvaise qu'une exploration de plusieurs temples fut entreprise à la recherche de la possibilité d'un meilleur enregistrement. Sans succès! Partout un jeu d'orgue qui couvrait plus qu'il ne guidait les voix et souvent un manque d'ensemble dans l'exécution des chants. Le fait est assez remarquable de la part d'un public le plus souvent amateur de musique et cultivé, et dans des temples où l'acoustique permet de donner des concerts. Un ethnologue protestant m'a un jour affirmé qu'il s'agissait là d'une ascèse, d'un puritanisme, qui refusait tout ce qui pourrait paraître enjolivement du culte, distraction du sens priant des paroles, par le plaisir esthétique. Il est vrai que cet argument est aussi donné, on l'a vu plus haut, pour fonder l'austérité du décor. Il arrive pourtant que certains chants, (particulièrement représentatifs de l'identité protestante?) soient entonnés de façon vibrante. Contre-exemples à propos de "Que Dieu se montre seulement» (Ps. 68) ou encore « À toi la gloire, O Ressuscité ! » qui infirment la thèse d'une indifférence ou d'une incapacité à maitriser de façon harmonieuse le chant d'assemblée. Il n'en reste pas moins qu'on a édifié là, en quelque sorte en creux ou en négatif, un autre contexte sensible qui contribue à la perception identitaire du protestantisme réformé français.

\section{Des variations à la sauvegarde du sens}

87 On peut être plus étonné par l'aspect processionnel dans le second exemple cité : les rameaux, les bougies des enfants évoquent une atmosphère catholicisante. En d'autres cas, on parlera de saynètes jouées par les enfants au cours du culte sur des thèmes bibliques. De telles expériences catéchétiques sont contestées. L'élimination de tous ces aspects cérémoniels, ou de spectacle, au temps de la Réforme avait été considérée comme le signe d'une foi adulte. Mais justement dans le cas des enfants dont on ne peut encore attendre pleinement ce caractère adulte, une plus grande ouverture à ces formes sensibles de l'initiation religieuse est-elle admise? S'agissant précisément d'enfants, on ne peut encore exiger ce caractère adulte. La prédication du dimanche des rameaux, lors d'un culte centré sur les enfants donne la réponse adaptée, en dépit du fait que la liturgie réformée ait longtemps rejeté presque totalement la notion de fêtes religieuses jugeant suspecte la sacralisation du calendrier qui peut s'en suivre.

La forme sensible centrale des rites cependant consiste dans la Cène. L'enjeu y est tel qu'elle a été et constitue toujours le point de rupture avec le catholicisme, mais aussi entre confessions protestantes ${ }^{19}$. Avec quoi et comment la consommation collective du pain et du vin met-elle en communion? Nous n'entrerons pas ici dans un débat que les siècles n'ont pas permis de résoudre mais nous dirons seulement que plusieurs conceptions coexistent au sein de l'Église Réformée elle-même, allant d'une conception de simple commémoration à celle d'une réelle présence du Christ qui se communique dans la communion, en passant par l'affirmation qu'il est présent de toute façon dans l'assemblée priante réunie en son nom. Les biens spirituels apportés par cette forme sensible sont en tous cas référés à l'écoute, dans le temps du même culte, de la Parole de Dieu qui se communique dans les lectures bibliques et la prédication, la communion elle-même étant précédée par l'écoute des paroles de l'Institution qui font écho à celles de la Cène du Christ. C'est la même parole de Dieu qui est communiquée des deux façons. Pour faire 
bref, un culte sans Cène est un culte qui se suffit, alors qu'une Cène ne peut se concevoir indépendamment d'une écoute biblique préalable.

Il y a donc très majoritairement chez les réformés, attachement et respect pour cette communication sensible, signe sous une autre forme de la grâce de Dieu, et crainte que la force de cette manifestation sensible ne prenne le pas sur la manifestation primordiale de la Parole. D'où, paradoxalement, étant donné l'importance de la Cène, fut-elle seconde, auxiliaire, c'est concernant ce moment qu'on va trouver le plus grand souci de « casser le rite » et cela de plusieurs manières :

- Dans l'évocation de l'institution, on évite tout ce qui peut apparaître comme un moment de consécration (à la différence des catholiques), les paroles « ceci est mon corps... mon sang... » ne sont accompagnées d'aucun geste, pas d'élévation du pain et des coupes qui puisse les désigner même à la simple vénération. Dans un second temps, on insiste sur le sens de partage qui est lié à la communion, le pain n'est pas consacré, il est rompu pour être distribué ; la coupe est juste saisie et présentée comme le signe de l'alliance. ${ }^{20}$

91 - Dans l'invitation aux participants on pratique la variété, la forme la plus recommandée consiste à faire cercle autour du pasteur, mais la disposition des temples, la place tenue par les bancs, le nombre des participants ne permettent que rarement cette forme souhaitable. Alors on multiplie les cercles successifs selon des ordres donnés au dernier moment par le pasteur ; on les étire tout autour du temple et de ses allées, ce qui n'a plus beaucoup à voir avec un cercle évocateur de communion ; en fin de compte on se résout dans les cas d'affluence extrême à faire passer le pain et les coupes dans les rangs en demandant à chacun de s'asseoir après la communion pour repérer les travées servies, ou encore dans certains grands rassemblements, on défile devant les pasteurs placés à des points fixes à l'instar des files de communiants catholiques. On peut par ailleurs avancer des valeurs symboliques ${ }^{21}$ à ces diverses pratiques : dimension communautaire du cercle, lien entre la prédication et la distribution par le pasteur, rôle célébrant de l'assemblée dans le passage de main en main...

92 - Il ne s'agit jamais d'azymes, mais si le cas le plus fréquent est actuellement le pain de mie coupé en dés, toutes sortes de pains peuvent être utilisées, voire des petits pains au lait qu'on se passe en les rompant au fur et à mesure. Anciennement la distribution était accompagnée de versets bibliques ${ }^{22}$, maintenant on écoute plutôt un chant ou l'orgue, ou comme dans le second exemple on manifeste un signe de communion avec son voisin. Les coupes peuvent être remplies de vin rouge ou de jus de raisin dans le cas des militants antialcooliques. Le pasteur ou un aide les présentent, une dans chaque main, aux communiants qui y boivent successivement. Elles ne sont presque jamais essuyées entre chaque personne comme le font les catholiques. Celui qui s'estimerait contagieux se contente d'élever la coupe en signe de respect en passant son tour. Dans cette crainte toutefois, on peut voir des cas où le vin est présenté dans des gobelets individuels, mais cette pratique, critiquée comme altérant le caractère communautaire, a peu d'adeptes ${ }^{23}$ et je ne l'ai pas rencontrée parmi les cas analysés.

93 C'est au moment de la Cène aussi qu'on peut remarquer une certaine dissonance entre cette attention des théologiens à éviter toute sacralisation et au contraire l'attitude des participants, paroissiens mais aussi parfois pasteurs, très concentrés, les regards qui s'évitent, la lenteur des gestes qui accompagnent la circulation de la coupe, l'embarras parfois dans l'exécution des consignes pour la circulation du pain (accepter le morceau tendu par le précédent tout en prenant le plat pour y saisir celui que l'on va tendre à son voisin en même temps que le plat et ainsi de suite... encore plus compliqué lorsque c'est 
un morceau à rompre qui circule...) et dont il serait mal venu de sourire. Il y a, à ce moment, un fort contraste entre le naturel d'une petite assemblée où tout le monde se connaît et le caractère compassé des gestes et des déplacements d'une part, les objectifs désacralisants et la sacralisation vécue par les participants d'autre part.

Chaque église locale est maîtresse non seulement de ses façons de communier mais de la fréquence avec laquelle la Cène accompagne le culte. Si on rappelle souvent que Luther et Calvin souhaitaient une célébration hebdomadaire de la Cène, si la pratique tend actuellement à s'en répandre, si le rythme ancien de quatre fois par an a presque disparu, l'ERF invite simplement les églises locales à la célébrer « normalement » une fois par mois ${ }^{24}$.

Cette relative rareté de la Cène maintient de façon sensible l'évidence de la prédication comme élément essentiel du culte. On a évoqué plus haut les enquêtes qui ont montré les risques de routinisation et de déplacement du sens reconnu à la prédication par les fidèles. Mes observations qui avaient pour objectif l'identification des formes sensibles des cultes ont peu porté sur le contenu de la prédication sinon pour observer, loin d'une routinisation, la grande liberté laissée aux pasteurs et aux communautés dans le choix des lectures bibliques et du thème des prédications. Il peut $\mathrm{y}$ avoir une ou plusieurs lectures bibliques; dans la plupart des temples, des Bibles sont placées sur les bancs pour que l'on puisse en suivre la lecture. Dans le contexte oecuménique actuel, les pasteurs peuvent suivre l'ordre des lectures sur trois ans adopté par les catholiques mais rien ne les y oblige comme rien n'oblige à adopter des lectures et des prédications en rapport avec un calendrier liturgique. Le premier exemple rapporté plus haut est celui d'un jour de Pentecôte où la prédication n'eut rien à voir avec l'évocation du don de l'Esprit. Si par contre dans un culte particulièrement imagé, le jour des Rameaux, en raison de la présence des enfants, le second exemple montre le cas d'une prédication en rapport avec la Cène et la Passion, il faut remarquer qu'on y chante déjà à trois reprises, à l'aube de l'évocation de la Semaine Sainte, le Christ ressuscité. C'est en effet sa perception comme actuellement vivant qui fait que l'on évoque sa passion et qu'on revit le partage de son dernier repas. Ce qui peut apparaitre au sens commun comme une rupture du cours de la commémoration est en fait ce qui manifeste de façon sensible le sens qu'ici on lui reconnaît.

L'observation d'un calendrier liturgique rythmant l'année par la commémoration des événements de l'Évangile était d'ailleurs aussi étrangère à Calvin qu'aux premiers temps de l'Église. Tous les dimanches se valent et tous commémorent la mort et la résurrection $\mathrm{du}$ Christ $^{25}$. C'est au cours du XXe siècle que la liturgie de l'Église réformée réorganise un calendrier liturgique avec proposition de cultes non seulement pour les principales fêtes mais pour les temps de l'Avent et du Carême ${ }^{26}$. Jean-Daniel Benoit suggérait même la restauration de couleurs liturgiques qui auraient pu orner le tapis de l'autel ou les signets de la Bible : violet pour le Carême et l'Avent, blanc pour Pâques et les grandes fêtes, rouge pour la Pentecôte et le dimanche de la Réformation, vert pour les autres temps. Je dois dire que je n'ai jamais rencontré cette utilisation ni dans les cultes dont j'ai conduit l'observation pour cette étude ni dans ceux auxquels j'ai assisté par ailleurs, sinon timidement dans la couleur des fiches d'ordre du culte glissées dans les recueils de chants. Mais surtout, sauf pour Noël et le cycle "semaine sainte-Pâques", je n'ai pas observé d'intérêt particulier des participants pour ce calendrier. Là aussi on rencontre donc une tension entre les objectifs ecclésiaux et la réception par l'assemblée. 
Mais ce n'est pas seulement par les variations manifestant la liberté voire la créativité des participants que se traduit le refus de voir le rite sombrer dans la fixité ou les minuties d'un ritualisme, c'est aussi dans les moindres aspects de la façon de le vivre. Une affaire de style! la simplicité, le rejet de tout caractère solennel, ce qui permet au pasteur du premier exemple d'interpeller en pleine célébration successivement les participants ou l'organiste pour adapter au fur et à mesure l'ordre et la durée des chants, d'avoir en chaire la carafe et le verre d'eau du conférencier, et de proposer le café en même temps que la bénédiction. Ce qui permet également dans le cas du second exemple de privilégier le pédagogique : on recommence l'entrée, on explique le danger des flammes en même temps que l'analogie du Christ et de la lumière...

\section{Un certain ordre du sensible}

manifestait une aspiration générale à la diversification des textes liturgiques ${ }^{28}$, des théologiens se sont intéressés aux aspects sensibles des cultes comme dialogue, aux gestes de la communion, à la longueur de la prédication ou de la distribution de la Cène, à l'introduction de moments de silence dans des célébrations traditionnellement axées sur la parole... à la restauration d'une visibilité du calendrier liturgique... Une réflexion est engagée sur l'investissement de la personne toute entière dans la Cène, l'importance des éléments matériels, pain et vin, des gestes, l'incorporation comme nourriture, le repas symbolique ne sont pas des analogies qui aident à la communication de la Parole, ils manifestent que l'adhésion chrétienne ne peut pas rester purement intellectuelle mais doit se traduire dans tous les aspects de la vie. Autant qu'une recherche d'oecuménisme, ne peut-on y reconnaître l'inscription dans une évolution culturelle générale qui requiert le sensible, « par rapport à l'ensemble du culte et à la prédication très cérébrale : les sens

Archives de sciences sociales des religions, 111 | juillet-septembre 2000 
sont mobilisés pour ce sacrement, l'ouie, la vue, le toucher, et le goût. La Cène répond ainsi à un besoin de vivre une liturgie avec la totalité de son être ${ }^{29}$ ».

Une commission liturgique créée en 1989 a étudié de nouvelles propositions qui ont été discutées et adoptées par les synodes régionaux de l'automne 1995 puis par le synode national qui s'est tenu à Mazamet en mai 1996 selon le processus en vigueur dans l'ERF. La presse en a rendu compte en mettant l'accent sur une citation du pasteur Michel Bertrand, président du Conseil national de l'ERF, «nous avions un sentiment d'éclatement... Il nous fallait définir un cadre de référence qui redonne une unité aux cultes de nos Églises. Tout en laissant la liberté de l'adapter. » C'est dire assez qu'il ne va pas s'agir d'une unité «à la romaine »!

En effet, la nouvelle liturgie prévoit cinq variantes principales de l'ordre du culte, dont une simplifiée pour les petites assemblées et une forme essentiellement organisée autour de citations bibliques. Quatre variantes comportent trois sous-variantes, culte sans célébration de la Cène, avec célébration de la Cène de type A (les personnes qui partagent la Cène ne sont invitées autour de la table que pour le temps de la communion) ou de type B (l'assemblée est invitée autour de la table pour toute la célébration de la Cène). Cela représente 13 variantes de culte dominical sans compter les propositions de liturgies pour les événements familiaux (mariages, décès...) Si on rappelle qu'il ne s'agit pas d'imposer ces formes, il y a encore un bel avenir pour la diversité.

Quels sont les agents de cette diversité ? Autrement dit : qui décide des formes du culte dans une église locale? qui décide du nombre des Cènes et du choix du type A ou B ? En théorie, le fonctionnement des églises de l'ERF associe largement les conseils presbytéraux et les pasteurs à toutes les décisions? On a pu noter pourtant que l'assemblée semble manifester une autre sensibilité que l'officiant et cela à des moments cruciaux : prédication ou Cène.

Dès les premiers temps de la Réforme, on l'a rappelé, la diversité a été justifiée par la volonté d'adapter la forme des rites à l'objectif majeur: permettre la meilleure compréhension et participation de tous. L'exemple souvent cité est celui de Calvin renonçant à imposer à Genève la pratique hebdomadaire de la Cène qu'il avait connue à Strasbourg et pour laquelle il disposait d'arguments théologiques, afin de respecter les formes qu'il avait trouvées à son arrivée. Encore actuellement, la diversité des cultes proposés par la nouvelle liturgie s'appuie explicitement sur le souci de correspondre à la diversité des sensibilités. Mais sous le terme de sensibilité, il apparait que l'on met des options théologiques : - rappeler la loi, ou la volonté de Dieu, avant ou après l'annonce de la grâce, - joindre ou séparer le récit de l'institution et le moment de la fractionélévation... En fait, la nouvelle liturgie prend peu en compte les formes sensibles des rites, à l'exception de l'attention portée aux positions debout ou assis... Le chant d'assemblée qui est une innovation essentielle de la Réforme et qui, par le patrimoine réuni et par ses modalités d'exécution, représente un lieu d'identification sensible des participants n'a pas été traité par la commission qui a préparé la réforme de la liturgie. L'hymnologie a été renvoyée à plus tard et à la préparation par une autre commission.

Laurent Gagnebin souligne que «la grande majorité des acteurs actuels du culte protestant, et principalement du côté réformé et français, ignorent tout de l'histoire récente des différentes recherches ou mouvements liturgiques et même de l'histoire du culte proprement $\operatorname{dit}^{30}$.» Ce manque d'intérêt est-il dû à l'indifférence ${ }^{31}$, à la pleine satisfaction de ce qui existe, ou à la certitude que chaque église peut apporter les changements souhaités selon sa convenance et donc n'a pas à se soucier outre mesure de 
recherches extérieures (suspectées de centralisme!). On pourrait voir une indication en ce sens dans le souhait largement exprimé par les synodes régionaux de voir publier le recueil des cultes de la nouvelle liturgie sous forme de classeur (comme l'ancienne Liturgie verte), ce qui rendrait plus facile le choix des types de séquences, alors que les rapporteurs du projet tiennent à la présentation par fascicule qui préserverait mieux la cohérence recherchée pour chaque culte.

Cela dit, il serait faux de prétendre opposer une sensibilité conservatrice des fidèles à une sensibilité novatrice des pasteurs ou des théologiens, en dépit du fait que la recherche de renouvellement des rites s'est rencontrée d'abord parmi les théologiens. Ce serait méconnaitre la pluralité des orientations chez les uns comme chez les autres. Et il faudrait savoir, même si l'importance ecclésiale en est différente, ce qui s'expérimente lors de cultes internes de mouvements ou de petits groupes pour évaluer les aspirations des laïcs qui là comme ailleurs s'expriment moins sur le plan théorique. Disons plutôt qu'il existe au moins deux orientations principales, non monolithiques, ni exclusives, d'attention aux formes sensibles des cultes: - l'une, pour une part inexprimée, mais massive, attachée à la sobriété des lieux et des gestes, aux psaumes et cantiques connus, aux formes traditionnelles de la prédication, tous traits rapportés d'ailleurs à des convictions théologiques autant qu'à des coutumes ou traditions, - l'autre, revendiquant la légitimité de formes de culte accordant plus au symbolique, à l'imagé, à ce qui passe par les sens, en particulier à une importance accrue accordée à la Cène, au calendrier liturgique, à l'élargissement du répertoire de chants, en se réclamant autant de l'expérience d'autres Églises que de la modernité. Mais il serait faux également de dire que la première privilégie un fonctionnement religieux identitaire car la capacité permanente d'adaptation et de renouvellement, de se réformer en permanence est également revendiquée comme protestante. Il serait faux aussi de prétendre au retour menaçant du sacré à travers le renouveau du rite car on en trouve également le risque dans certaines façons d'effectuer les pratiques traditionnelles. Les aspects sensibles de la religion se rencontrent donc bien en dépit des idées reçues au sein des rites réformés et bien antérieurement aux désirs de renouvellement de la liturgie, et ces projets les mettent désormais au cœur du débat.

\section{BIBLIOGRAPHIE}

BENOIT Jean-Daniel, Initiation à la liturgie de l'Église Réformée de France, Paris, Berger-Levrault, 1956.

CARBONNIER-BURKARD Marianne, «Jours de fête dans les Églises Réformées de France au XVII ${ }^{\mathrm{e}}$

siècle. ", Études théologiques et religieuses, 1993, n 3, pp. 347-358.

GAGNEBIN Laurent, Le culte à chœur ouvert, Introduction à la liturgie du culte réformé, Paris, Les

Bergers et les Mages, Genève, Labor et Fides, 1992.

GoUNELLE André, « Le Culte selon la tradition réformée », Information-Évangélisation, 1988, n 1, pp. 2-13.

Gounelle André, La Cène, sacrement de la division, Paris, Les Bergers et les Mages, 1996. 
GRELLIER Isabelle, «Attentes à l'égard de la prédication et écoute de celle-ci dans diverses paroisses alsaciennes », Foi et Vie, n²-3, avril 1986.

MARTI F., BABUT E., « La prédication vue par ceux qui la reçoivent », Information-Evangélisation, n - 2, 1990.

PAStoureau Michel, « Les couleurs de l'art protestant », Libre-Sens, n 57, juillet-août 1996, pp. 192-195.

REYMOND Bernard, Liturgies en chantier, Lausanne, Éditions Belle Rivière, 1984.

REYMOND Bernard, L'architecture religieuse des protestants, Genève, Labor et Fides, 1996.

Roussel Bernard, «Faire la Cène » dans les églises réformées du royaume de France au seizième siècle (ca 1555-ca 1575), Archives de sciences sociales des religions, n 85, 1994, pp. 99-119.

SCHLEMMER André, En Esprit et en Vérité, Le culte dans l'Église Réformée, Paris, Messageries Évangéliques, 1947.

WiLlAime Jean-Paul, « Prédication, culte protestant et mutations contemporaines du religieux », Foi et Vie, $\mathrm{n}^{\circ} 2-3,1986$, pp. 7-21.

WiLlAime Jean-Paul, La Précarité protestante, Genève, Labor et Fides, 1992.

Sociologie de la Liturgie, Social Compass, $\mathrm{n}^{\circ}$ 2, 1975.

LXXXIX Synode national, Église Réformée de France, Mazamet, 16-19 mai 1996, Titre V, pp. 563-727.

\section{NOTES}

1. Elle fut précédée par une liturgie, de Calvin également, pour l'Église française de Strasbourg dont aucun exemplaire n'a été conservé.

2. ROUSSEL, 1994.

3. On en trouvera les références en bibliographie annexe.

4. En réalité, la variété dans l'Église catholique a été plus importante et plus constante que la théorie ne le présente, mais nous ne discuterons pas ici du bien fondé de cette perception.

5. Je ne traiterai pas ici du baptême en tant que tel mais seulement de ce qui concerne sa place dans l'ordonnance du culte.

6. WiLlaime, 1992.

7. Elle fut précédée par une liturgie, de Calvin également, pour l'Église française de Strasbourg dont aucun exemplaire n'a été conservé.

8. Je ne traiterai pas ici du baptême en tant que tel mais seulement de ce qui concerne sa place dans l'ordonnance du culte.

9. En réalité, la variété dans l'Église catholique a été plus importante et plus constante que la théorie ne le présente, mais nous ne discuterons pas ici du bien fondé de cette perception.

10. PASTOUREAU, 1996.

11. REYMOND, 1984, 1996.

12. Pastoureau, 1996.

13. REYMOND, op. cit... 1996.

14. Les musées du protestantisme présentent généralement des exemples conservés de telles chaires démontables qui étaient utilisées pour les cultes clandestins au temps des persécutions. Elles témoignent de l'importance symbolique de cette disposition.

15. Willaime, 1992. 
16. Grellier, 1986 et MARTi, Babut, 1990.

17. LXXXIXe Synode national, 1996.

18. Actuellement on recense trois recueils principaux de cantiques : Louange et prière (1939), Nos coeurs te chantent (1979), Arc-en-ciel (1986). L'adoption de chacun d'eux est ou a été l'objet de débats passionnés dans les paroisses.

19. GounelLe, 1996.

20. La nouvelle liturgie commune de l'ERF ne maintient pas cette dissociation entre l'évocation de l'institution et le moment de la fraction-élévation pour les cultes de type B.

21. Gounelle, op. cit.

22. Recueil de textes liturgiques de l'ERF, dit Liturgie Verte en raison de sa couverture, BERGERLEVRAULT, 1963. Il y était recommandé d'accompagner la distribution par les phrases « ceci est mon corps donné pour toi », « ceci est mon sang versé pour toi » avec toutefois une préférence pour une distribution silencieuse.

23. «On sent une contradiction pénible entre l'acte de fraternité chrétienne qui affirme l'unité parfaite du corps du Christ et la séparation intime des chrétiens les uns des autres, ou même le dégoût du voisin », SCHLEMMER, 1947.

24. Art. 7 de la Discipline de l'ERF.

25. C'est seulement à partir du XVIII ${ }^{e}$ siècle que la liturgie de Genève qui, après le temps du Désert, va être adoptée en France, réintroduit des célébrations annuelles : Noël (1724), puis le Vendredi saint (1828), plus tardivement Pâques et la Pentecôte (1861). CARBONNIER-BURKARD, 1993. L'auteur évoque la concession de prédications les jours de fêtes que les protestants français étaient contraints de chômer selon les prescriptions catholiques. Cette concession visait surtout à les préserver de la tentation de se joindre aux fêtes catholiques, Noël en particulier.

26. BENOIT, 1956.

27. Synode national d'Orthez, 1963. "Nous nous réjouissons d'accueillir à la communion du Seigneur tous ceux qui, membres ou non de notre Église, veulent s'en approcher en discernant dans le pain et le vin, le corps et le sang du Christ. »

28. BENOIT, op.cit., «À la suite d'une vaste enquête menée auprès des Églises et de leurs conseils presbytéraux, il s'est avéré qu'on demandait encore et toujours plus de variantes ».

29. GAGNEBIN, 1997.

30. Ibid.

31. BENOIT, op. cit.La liturgie étant une prière qui ne dit pas «je » mais «nous ", il attribue le manque d'intérêt pour la liturgie à l'individualisme protestant.

\section{RÉSUMÉS}

Une idée reçue voudrait que rien ne fut concédé au sensible dans l'ordonnance traditionnelle des cultes réformés. L'observation méthodique de 27 cultes dans 5 régions différentes de l'Église Réformée de France, dont on détaille ici deux exemples, met en évidence d'une part, malgré une apparence de grande diversité de certains choix liturgiques, des éléments majoritairement présents dont la perception constitue de fait un conditionnement sensible fort : clarté, nudité du décor, corpus et modalité d'exécution des chants, chaire et robe pastorale... et nombre de traits plus ou moins récents et dont l'adoption n'est ni universelle ni sans tensions : fréquence de la Cène, calendrier liturgique, élargissement du patrimoine musical... Ces évolutions résultent de la 
recherche explicite par les partisans d'un renouveau liturgique de faire plus de place au symbolique, à l'imagé, à ce qui passe par les sens, en se réclamant autant de l'expérience d'autres Églises que de la modernité. Le sensible est bien présent dans les cultes réformés, il est même actuellement au cœur du débat.

It is generally admitted that no attention should be given to sensitivity in the traditional pattern of Reformed worship. A survey of 27 services of the French Reformed Church in 5 different regions, two of which are reported in details here, highlights on the one hand - despite the great diversity of certain liturgical choices -, the overwhelming presence of elements whose perception represents a strong attention to the sensitive: the light, the bareness of the setting, the choice and the performance of the songs, the pulpit and the pastoral dress... together with a number of more or less recent features whose introduction is neither universal nor without tension: the frequency of the Lord's Supper, the liturgical calendar, the broadening of the musical references... This evolution is the resuit of an explicit attempt, on the part of the advocates of liturgical renewal, to make more room for symbolism, imagery, for what is perceptible by the senses. This attempt takes its inspiration from the experience of other churches as much as from modernity. The sensitive is therefore present in the reformed services. More than that, it is currently at the center of the discussion.

Una idea muy expandida sobre los cultos reformados insiste en el hecho de que nada fue concedido a lo sensible en la ordenación tradicional de estos cultos. Por lo tanto, la observación de 27 cultos en 5 regiones de la Iglesia Reformada de Francia, de los cuales la autora destaca dos ejemplos en este artículo, implica matizar el propósito. En efecto, pone en evidencia que, a pesar de una apariencia de gran variedad de algunas opciones litúrgicas, existen en la mayoría elementos cuya percepción constituye de hecho un condicionamiento fuerte de lo sensible: claridad, despojamiento ornamental, corpus y modalidad de ejecución de los cantos, cátedra sagrada y traje pastoral... Además, y en este sentido, la observación evidencia numerosos rasgos que son más o menos recientes, y cuya adopción no se hace de manera universal ni siquiera sin tensiones: frecuencia de la Cena, calendario litúrgico, desarrollo del patrimonio musical, etc. Estas evoluciones resultan de la búsqueda explicita de los partidarios de una renovatión litúrgica, que tratan de dar más espacio a lo simbólico, a la imagen, a lo que pasa por los sentidos, apelando tanto a la experiencia de otras Iglesias como de la modernidad. Evoluciones que muestran que, efectivamente, lo sensible esta presente en los cultos reformados, y más, que ocupa actualmente el centro del debate.

\section{AUTEUR}

\section{FRANÇOISE LAUTMAN}

Groupe de sociologie des religions et de la laïcité 